\title{
Time Series Calculation of Heart Rate Using Multi Rate FIR Filters
}

\author{
MR Risk ${ }^{1}$, DF Slezak ${ }^{1}$, P Turjanski $^{1}$, A Panelli $^{1}$, RAM Taborda $^{2}$, G Marshall $^{1}$ \\ ${ }^{1}$ CONICET and Computer Science Department, FCEyN University of Buenos Aires, Argentina \\ ${ }^{2}$ LIADE, FCEFyN National University of Córdoba, Argentina
}

\begin{abstract}
The spectral analysis of heart rate variability, based on the Fourier transform, needs even sampled data. The objectives of this study were to develop an interpolation method based on multi rate FIR filters, and then to implement this method for parallel processing machines. A total of three data sets were used: a) simulated heart rate with an IPFM model, b) autonomic blockage database (both pharmacological and postural), and c) long term Holter studies (recordings of 24 hours). Spectral analysis, for the three data sets, was processed for both interpolation using FIR filters and cubic splines, the results for Bland and Altman analysis for low frequency band, showed a difference of $-47 \pm 131 \mathrm{~ms}^{2}$; then for the high frequency band, the difference was $3 \pm 48 \mathrm{~ms}^{2}$. The presented method of time series calculation, using FIR filters, probed to be equivalent for both simulated and real data, and is suitable for parallel programming implementation.
\end{abstract}

\section{Introduction}

Time series is a method to represent a variable in the course of time, such as distance, speed, or any other physical variable, versus time, as well currency prices or other econometric variable, and physiological variables too; in other words the applications of time series range across science, engineering, economics and medicine, among others. The evenly sampled time series are necessary if the next step is a method such as the Fourier transform, or other spectral estimation methods based on the Fourier transform, which needs by definition evenly sampled source data.

Many times, the time series volume of data is very high. Processing this huge amount of data requires high performance computing (HPC) techniques. One of the most usual way of processing this is parallelizing the application and processing the files in beowulf computer clusters [1].

Nowadays, many organization collaborate in one unique huge project which may consist in distributed resources over the world. In this context, in which the resources from different institutions must be shared in an efficient way,
Grid Computing emerges as the most promising technology to enable collaboration among organizations regardless of their geographically location [2].

Among the many applications of high performance computing, one of the biomedical applications is the study of the autonomic nervous system (ANS) through the heart rate variability (HRV).

The HRV provides a non invasive window to the ANS; the ANS controls a number of viscerae, such as the heart, lungs, arteries and veins, glands, among others, and according its name, this underlining controls is automatic, without any consciuos component [3]. In presence of diseases as diabetes the ANS may be impaired, leading to a lack of proper control [4].

The aim of this paper is to present a method of calculation of evenly spaced time series, using finite response filters (FIR), and implemented in parallel processing (Beowulf cluster), and Grid-based computing system.

\section{Study groups}

A total of three data sets were used: a) simulated heart rate, b) autonomic blockage database, and c) long term Holter studies. The simulated heart rate used an integral pulse frequency modulation (IPFM) model, the heart rate was modulated with two sines of 0.1 and $0.25 \mathrm{~Hz}$; the autonomic blockage database has data from 12 healthy subjects, with a combination of postural (supine and standing) and pharmacological (atropine and propranolol) conditions; and the long term database used 10 Holter recordings of 24 hours each.

The first and second studies were used to compare the two methods of interpolation, using cubic splines and FIR filters; the third study was used to process the ECG beat series with parallel and GRID computing.

\section{Heart rate simulation}

One of most widely used HR simulator is the IPFM model [5]. In the IPFM model, where the input signal $x(t)$ pass through an integrator, then generating the ouput $y i(t)$, entering this output to a comparator input, and the other 
input of this comparator is connected to a treshold $V_{T}$, the output of the comparator is used to reset the integrator, and is finally the output of the model.

The output of the integrator is modelized with the following equations (1) and (2):

$$
\begin{aligned}
& y i(t)=\frac{1}{x_{k}} \int_{t_{k}}^{t} d t \quad \text { if } y i(t)<V_{T} \quad t_{k} \leq t<t_{k+1}(1) \\
& y i(t)=\quad 0 \quad \text { if } y i(t)=V_{T} \quad t=t_{k+1}
\end{aligned}
$$

From equations (1) and (2):

$$
V_{T}=\frac{1}{x_{k}} \int_{t_{k}}^{t} d t=\frac{t_{k+1}-t_{k}}{x_{k}}=\frac{\tau_{k}}{x_{k}}
$$

where $t_{k+1}-t_{k}$ is the interval between two consecutive beats.

From equation (3), we get equation (4) which is the beat to beat HR simulation:

$$
y(t)=\sum_{k} \delta\left(t-t_{k}\right)
$$

The figure 1 shows a simulation run, where $x(t)=x_{0}+$ $A_{0} \operatorname{sen}\left(f_{0} 2 \pi t\right)+A_{1} \operatorname{sen}\left(f_{1} 2 \pi t\right), f_{0}=0.1 \mathrm{~Hz}$ and $f_{1}=$ $0.25 \mathrm{~Hz}$, and $V_{T}=1000 \mathrm{~ms}$.
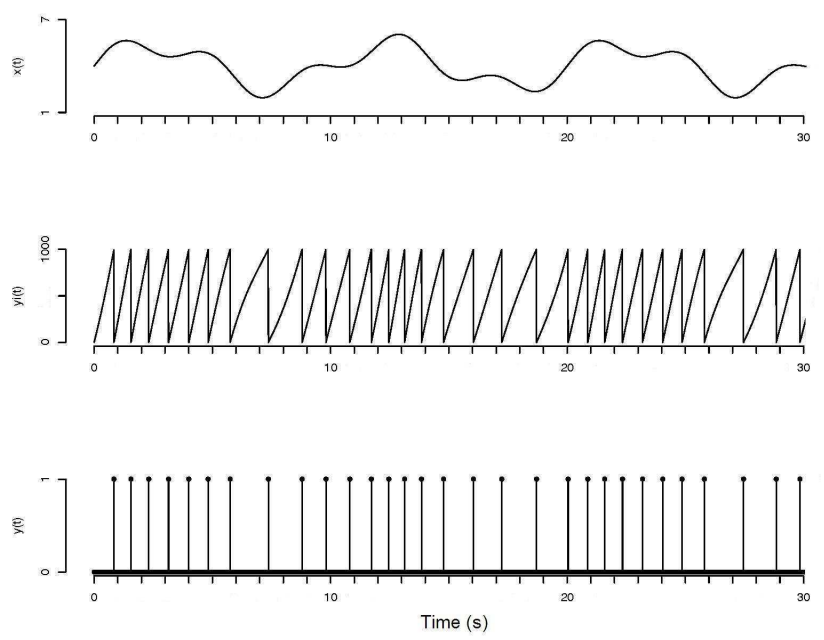

Figure 1. Signals of the IPFM model for a combination of $0.1 \mathrm{~Hz}$ and $0.25 \mathrm{~Hz}$ sine waves.

The figure 2 shows the simulated beat series, with the above described modulation.

\section{Interpolation using FIR filters}

The interpolation using FIR filters has been used in a number of processing methods, such as voice processing,

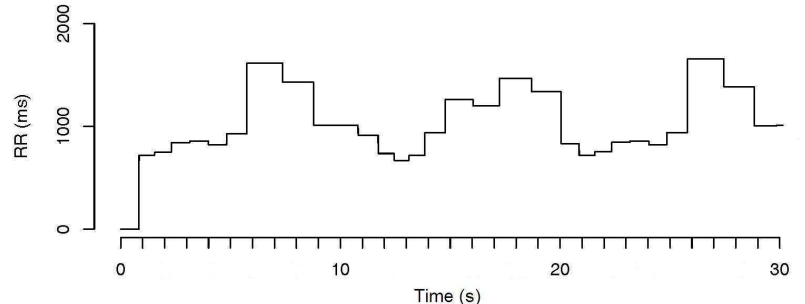

Figure 2. Beat series simulated, with two sine waves modulation.

and usually the design of the filters optimizes phase distortion [6]. The interpolation process using FIR filters can be considered as a multirate processing [7], in which is combined filtering with changes in sampling frequency, called decimations.

In applications where is required big changes of sampling frequency, multiple stages are used splitting the change in sampling frequency, in example for a total reduction $M=M_{1} M_{2} \ldots M_{I}$, uses $I$ stages. Each stage has a low pass filter and then the decimation; the filtering before decimation is necessary to minimizes the aliasing. FIR filters are widely used in interpolation because are more stable and has linear phase [7].

The figure 3 shows three stages, which reduce the original sampling frequency from 250 to 5 samples per second; beat series enter to the first stage at the original ECG sampling rate, then the low pass filter (LPF1) preceeds the first stage of decimation which decimates by 2 . The same figure 3 shows the two following stages, LPF2 and LPF3, preceeded by decimation stages each one by 5 , finally the sampling rate is 5 samples per second.

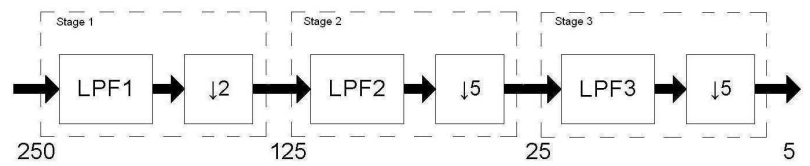

Figure 3. Block diagram of the interpolation process using FIR filters and decimation.

The coefficients of the low pass filters were calculated with the Remez method [8], which allows the design of optimized Chebyshev filters. The equation to calculate the number of coefficients, for a moderate width of the pass band is:

$$
N=1+\frac{-20 \log \sqrt{\delta_{1} \delta_{2}}-13}{14.6\left(f_{s}-f_{p}\right)}
$$

where $\delta_{1}$ determines the maximum ripple of the pass band, $\delta_{2}$ determines the attenuation in the attenuation 
band, $f_{s}$ and $f_{p}$ are the cutoff and attenuation normalize frequencies respectively.

In the present work $\delta_{1}=0.025$, and $\delta_{2}=0.001$, then we obtain an attenuation of $-60 \mathrm{~dB}$. Using equation (5) the number of coefficients is $N=1132$ if we use just one stage of decimation, with cutoff frequencies of $f_{s}=$ $2 / 250$ and $f_{p}=1.5 / 250$, this calculation shows clearly the necessity of the use of multiple stages.

The cutoff frequencies where $f_{s}=100 / 250$ and $f_{p}=$ $5 / 250$ for the first stage, then $f_{s}=20 / 125$ and $f_{p}=$ $5 / 125$ for the second stage, and finally $f_{s}=4 / 25$ and $f_{p}=1.5 / 25$ for the third stage, where the number of coefficients were $N 1=7, N 2=21$ and $N 3=25$, for first, second and third stage respectively. The figure 4 shows the frequency response for each filter.

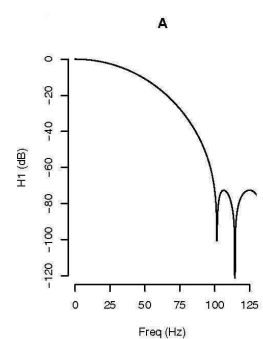

Freq $(\mathrm{Hz})$

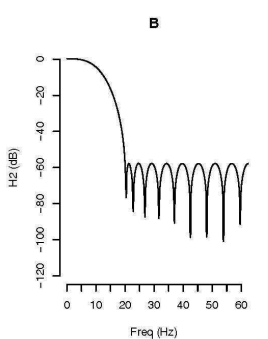

Freq (Hz)

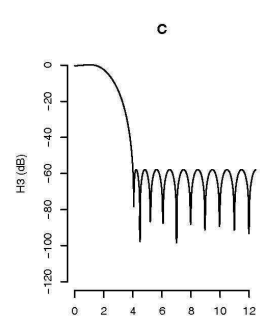

Freq $(\mathrm{Hz})$
Figure 4. Frequency response for each low pass filter of the multirate interpolator, where panels A, B and C correspond to filters LPF1, LPF2 and LPF3 respectively.

\section{Results}

\subsection{Simulated data}

The simulated beat series are processed with two interpolation methods; the cubic splines method trends to interpolate with values which overestimate the RR intervals, in special for the local maximums. This overestimation may lead to overestimation in the frequency domain; however is barely shown in the power spectrum density (PSD). The PSD was calculated using the Blackman-Tukey method, which is a Fourier based method with a Gaussian window [9].

The spectral analysis of the simulated HR, showed a miscalibration in frequency around the peaks, at 0.1 and $0.25 \mathrm{~Hz}$. In the case of the FIR filter method, the maximum for the $0.1 \mathrm{~Hz}$ oscillation was $754032 \mathrm{~ms}^{2} / \mathrm{Hz}$ located at $0.100098 \mathrm{~Hz}$; then for the $0.25 \mathrm{~Hz}$ oscillation was $151243 \mathrm{~ms}^{2} / \mathrm{Hz}$ at $0.246582 \mathrm{~Hz}$. On the other side, using the cubic splines method, the maximum for $0.1 \mathrm{~Hz}$ oscillation was $773225 \mathrm{~ms}^{2} / \mathrm{Hz}$ at $0.100098 \mathrm{~Hz}$; for the $0.25 \mathrm{~Hz}$ oscillation was $131310 \mathrm{~ms}^{2} / \mathrm{Hz}$ at $0.25146 \mathrm{~Hz}$.

The energies for low frequency band (LF, 0.04 to 0.15 $\mathrm{Hz}$ ) and high frequency ( $\mathrm{HF}, 0.15$ to $0.4 \mathrm{~Hz}$ ), in agree- ment with standard autonomic studies $[9,10]$, using the FIR method were 40603 and $9828 \mathrm{~ms}^{2}$, respectively. The cubic splines method showed for LF an energy of 40327 $\mathrm{ms}^{2}$, and for HF was $11057 \mathrm{~ms}^{2}$. The correlation between each method, between 0.04 and $0.4 \mathrm{~Hz}$, was 0.993 ; the spectral resolution was $0.00244 \mathrm{~Hz}$. A regression analysis showed an intercept of $2851 \mathrm{~ms}^{2} / \mathrm{Hz}(P=0.001)$ and the slope was $0.963(P<0.0001)$; the determination index was $r^{2}=0.9929$. The distribution of the residuals was verified to have a normal distribution, using the Shapiro-Wilk test [11].

\subsection{Autonomic blockage database}

The figures 5 and 6 shows the time series and the PSD, for each method, in two representative recordings CRC03STC and CRC07SUA, respectively.

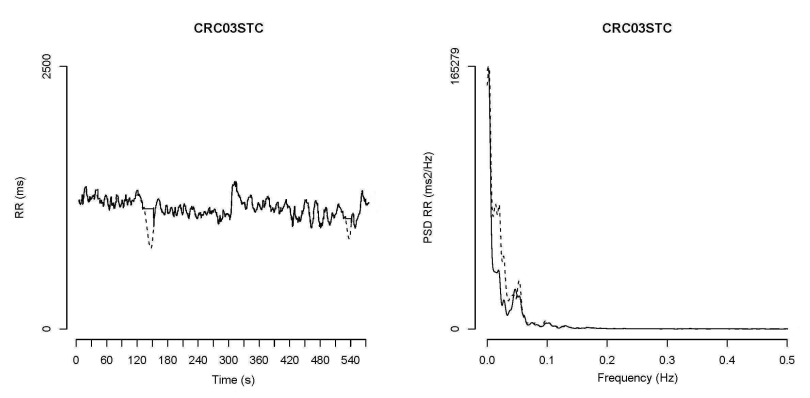

Figure 5. Time series and PSD of recording CRC03STC.

The figure 5 shows in the left panel the time series, for both interpolation methods, and as is shown how sometimes the cubic splines method overestimates the excursion of the time series (dashed line), then this overestimation is present in the PSD at low frequencies, as is shown in the right panel of the same figure 5. The figure 6 shows the time series and PSD for recording CRC07SUA, in this case the two methods are similar.
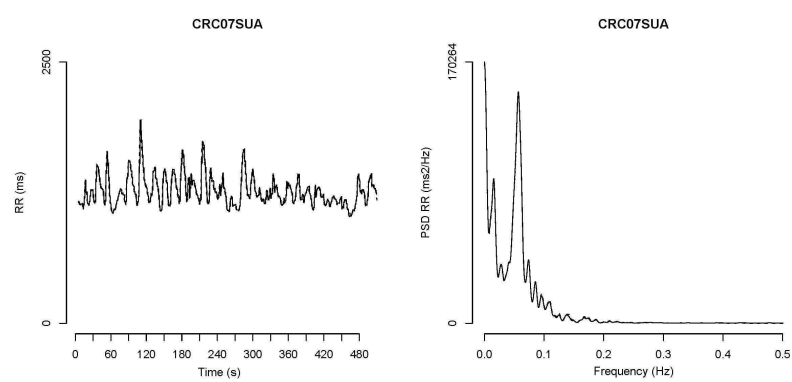

Figure 6. Time series and PSD of recording CRC07SUA.

The analysis of Bland-Altman [12], allows the comparison of two diagnostic methods; in the present study it was used to compare the LF and HF energies from 
the two interpolation methods. The mean difference at LF energies was $-46.5 \pm 131.3 \mathrm{~ms}^{2}$, the regression analysis in the Bland-Altman plot shows a significant slope of $-0.031(P=0.0002)$, with a coefficient of determination $r^{2}=0.16$. The $95 \%$ of the differences, or in other words the $\pm 2 S D$ around the mean of the differences, were between the range of $525 \mathrm{~ms}^{2}$.

Finally the difference at HF energies was $3 \pm 48 \mathrm{~ms}^{2}$, the regression analysis in the Bland-Altman plot shows a significant slope of $0.06(P<0.0001)$, with a coefficient of determination $r^{2}=0.27$. The $95 \%$ of the differences, or in other words the $\pm 2 S D$, were between the range of 192 $m s^{2}$.

\section{Discussion}

In the present work two methods for the calculation of evenly spaced time series from unevenly spaced beat series were compared, cubic splines and multi rate FIR methods. The cubic splines method, which is widely used in most applications, specially in the case of HRV studies [13]; other studies used linear interpolation [14][9].

The new method presented in this paper, using FIR filters and decimation, is equivalent to previously used methods for the calculation of time series. The analysis of simulated beat series with the IPFM model, showed that the FIR filter method has a more accurate frequency calibration, this may be because the nature of method, using decimation, leads to a more accurate sampling rate of the time series; on the other side the cubic splines method may introduce rounding errors leading to a frequency miscalibration.

The comparison of the two methods using the database of autonomic blockage showed differences between the two methods, according to the results of the Bland-Altman analysis for both LF and HF bands, however this differences are acceptable for medical clinical applications [12].

Finally, we developed parallel and grid implementations for processing evenly spaced time series, using multi rate FIR filters. The parallel application developed shows a clear performance improvement versus R-prototype and serial implementations. The Grid application allows processing many time series files simultaneously, increasing even more the performance. Although the performance was augmented, ECG standard files do not need strictly HPC, as they can be processed each one, in a few minutes. Nevertheless, the use of HPC and grid computing in this area would permit the implementation of more complex algorithms for the analysis of ECG.

\section{Acknowledgements}

The authors acknowledge Microsoft Research for its support. Marcelo Risk and Guillermo Marshall are inves- tigators of the National Council for Scientific and Technological Research (CONICET), Argentina.

\section{References}

[1] Pacheco PS. Parallel programming with MPI. San Francisco, CA, USA: Morgan Kaufmann Publishers Inc., 1996. ISBN 1-55860-339-5.

[2] Foster I, Kesselman C, Nick J, S. T. The physiology of the grid: An open grid services architecture for distributed systems integration. In Global Grid Forum. 2002; .

[3] Guyton A, Hall J. Texbook of Medical Physiology. W.B. Saunders Company, 2005.

[4] Risk M, Bril V, Broadbridge C, Cohen A. Heart rate variability measurement in diabetic neuropathy: Review of methods. Diabetes Technology and Therapeutics 2001; 3(1):63-76.

[5] Yang F, Liao W. Modeling and decomposition of hrv signals with wavelet transform. IEEE Engineering in Medicine and Biology July/August 1997;17-22.

[6] Schafer R, Rabiner L. A digital signal processing approach to interpolation. Proc IEEE 1973;61:156-166.

[7] Ifeachor E, Jervis B. Digital signal processing: a practical approach. Addison-Wesley, 1993.

[8] Rorabaugh C. Digital filter designer's hadnbook with C++ algorithms. McGraw-Hill, 1997.

[9] Risk M, Sobh J, Barbieri R, Armentano R, Ramírez A, Saul J. Variabilidad de las señales cardio-respiratorias. parte 1: Variabilidad a corto plazo. Revista Argentina de Bioingeniera 1996;2(1).

[10] Saul J. Beat to beat variations of heart rate reflect modulation of cardiac autonomic flow. NIPS 1990;5.

[11] Venables W, Smith D, the R development core team. An introduction to $\mathrm{R}$, notes on $\mathrm{R}$ : a programming environment for data analysis and graphics, version 2.1.1. R foundation, 2005.

[12] Bland J, Altman D. Statistical methods for assessing agreement between two methods of clinical measurement. Lancet 1986;i:307-310.

[13] Rompelman O, Coenen A, Kitney R. Measurement of heart-rate variability: Part 1 - comparartive study of heartrate variability analysis methods. Medical and Biological Engineering and Computing May 1977;233-239.

[14] Berger R, Akselrod S, Gordon D, Cohen R. An efficient algorithm for spectral analysis of heart rate variability. IEEE Transactions on Biomedical Engineering 1986; BME-33(9):900-904.

Address for correspondence:

Marcelo Risk, Ph.D.

Department of Computer Science

FCEyN, Universidad de Buenos Aires

Pabellón I - Ciudad Universitaria (C1428EGA)

Buenos Aires - Argentina

mrisk@dc.uba.ar 